\title{
On Some Trigonometric Integrals
}

\author{
By Henry E. Fettis
}

Abstract. Expressions are obtained for the integrals

$$
I_{\lambda}^{(p)}=\int_{0}^{\pi / 2}\left(\frac{\sin \lambda \theta}{\sin \theta}\right)^{p} d \theta, \quad J_{\lambda}^{(p)}=\int_{0}^{\pi / 2}\left(\frac{1-\cos \lambda \theta}{\sin \theta}\right)^{p} d \theta
$$

for arbitrary real values of " $\lambda$ ", and $p=1,2$.

1. The integrals

$$
I_{\lambda}^{(p)}=\int_{0}^{\pi / 2}\left(\frac{\sin \lambda \theta}{\sin \theta}\right)^{p} d \theta
$$

and

$$
J_{\lambda}^{(p)}=\int_{0}^{\pi / 2}\left(\frac{1-\cos \lambda \theta}{\sin \theta}\right)^{p} d \theta, \quad p=1,2,
$$

are of a sufficiently general, standard type that one would expect to find them in almost any table of integrals or other reference work (e.g., [1], [2] , [3]). However, a comprehensive search by the author has disclosed that (with the exception of (1) for integer " $\lambda$ "), these integrals are conspicuously absent from the literature. Should any one of the above integrals arise in a practical problem (as well they might) one would, in the absence of a closed-form expression, be inclined to evaluate it either numerically or in series. However, as will be shown in the following sections, this is not necessary, as such a closed form does exist, and can in fact be found in terms of the logarithmic derivative of the gamma function, $\Psi(z)$.

2. It is evident that $I_{\lambda}^{(1)}$ and $J_{\lambda}^{(1)}$ satisfy the recurrence relations

$$
\begin{aligned}
& I_{\lambda+1}^{(1)}-I_{\lambda-1}^{(1)}=\frac{2}{\lambda} \sin \frac{\pi}{2} \lambda, \\
& J_{\lambda+1}^{(1)}-J_{\lambda-1}^{(1)}=\frac{2}{\lambda}\left(1-\cos \frac{\pi}{2} \lambda\right),
\end{aligned}
$$

with $I_{0}^{(1)}=J_{0}^{(1)}=0, I_{1}^{(1)}=\pi / 2, J_{1}^{(1)}=\ln (2)$, from which we easily obtain, by induction, that for integer values of $\lambda=n>0$,

$$
I_{2 n}^{(1)}=2 \sum_{k=0}^{n-1} \frac{(-1)^{k}}{(2 k+1)}, \quad I_{2 n+1}^{(1)}=\frac{\pi}{2},
$$

Received February 5, 1980.

1980 Mathematics Subject Classification. Primary 33A 15, 33A10.

Key words and phrases. Integrals, definite integrals, trigonometric integrals, Gamma function, Psi function. 
(6) $J_{2 n}^{(1)}=2 \sum_{k=0}^{n-1} \frac{1}{(2 k+1)}, \quad J_{4 n-1}^{(1)}=J_{4 n+1}^{(1)}=\ln (2)+2 \sum_{k=0}^{n-1} \frac{1}{(2 k+1)}$.

The results (5) are well known (see [1, Eqs. 3.612-3]), while those of (6) have been previously found by the author (Math. Mag., v. 39, no. 5, 1966, p. 281) but do not appear in any of the standard references, such as [1], [2], [3].

3. For $p=1$ and noninteger values of " $\lambda$ ", the integrals (1) and (2) can be readily evaluated by complex integration of the function

$$
f(z)=\frac{1-z^{\lambda}}{z^{2}-1}
$$

in the $z=\rho e^{i \theta}$ plane around the contour consisting of

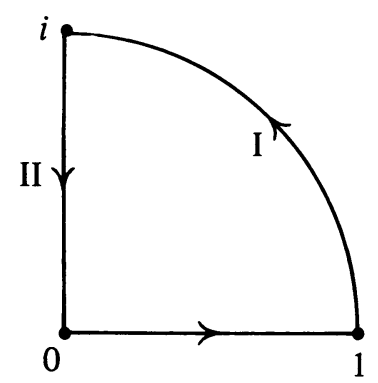

I-The quadrant of the unit circle $z=e^{i \theta}$ $(0 \leqslant \theta \leqslant \pi / 2)$.

II-The imaginary axis extending from $z=i$ to $z=0$, and the real axis from $z=0$ to $z=1$.

The integral around this contour must be zero, since the integrand has no singularities within it, and is single valued throughout.

On the arc constituting part I of the contour, $z=e^{i \theta}$, and integration gives

$$
i \int_{0}^{\pi / 2} \frac{1-e^{i \lambda \theta}}{e^{i \theta}-e^{-i \theta}} d \theta=\frac{1}{2} \int_{0}^{\pi / 2} \frac{1-e^{i \lambda \theta}}{\sin \theta} d \theta=\frac{1}{2}\left(J_{\lambda}^{(1)}-i I_{\lambda}^{(1)}\right)
$$

On the portion from $z=i$ to $z=0, z=y e^{i \pi / 2}(0 \leqslant y \leqslant 1)$, and hence integration along this segment gives

$$
+e^{i \pi / 2} \int_{0}^{1} \frac{1-e^{i \pi \lambda / 2} y^{\lambda}}{1+y^{2}} d y=\frac{1}{2} e^{i \pi / 2} \int_{0}^{1} \frac{t^{-1 / 2}\left(1-e^{i \pi \lambda / 2} t^{\lambda / 2}\right)}{1+t} d t
$$

while on the segment from $x=0$ to $x=1$, the contribution is

$$
\int_{0}^{1} \frac{1-x^{\lambda}}{x^{2}-1} d x=-\frac{1}{2} \int_{0}^{1} \frac{t^{-1 / 2}\left(1-t^{\lambda / 2}\right)}{1-t} d t
$$

Both of the integrals (9) and (10) can be expressed in terms of the function $\Psi(z)=$ $d[\ln \Gamma(z)] / d z$, using formulae 5 and 6 from [2, Chapter I, p. 9]. Finally, when the total integral is set equal to zero, and real and imaginary parts separated, we obtain

$$
\begin{aligned}
& J_{\lambda}^{(1)}=\Psi\left(\frac{\lambda+1}{2}\right)-\Psi\left(\frac{1}{2}\right)-\frac{1}{2} \sin \left(\frac{\pi}{2} \lambda\right)\left[\Psi\left(\frac{\lambda+3}{4}\right)-\Psi\left(\frac{\lambda+1}{4}\right)\right], \\
& I_{\lambda}^{(1)}=\frac{\pi}{2}-\frac{1}{2} \cos \left(\frac{\pi}{2} \lambda\right)\left[\Psi\left(\frac{\lambda+3}{4}\right)-\Psi\left(\frac{\lambda+1}{4}\right)\right] .
\end{aligned}
$$


4. The results (11) can be found alternatively in the following way. We have

$$
\begin{aligned}
I_{\lambda+1}^{(1)}+I_{\lambda-1}^{(1)} & =2 \int_{0}^{\pi / 2} \sin \lambda \theta \cot \theta d \theta=\int_{0}^{\pi} \sin \frac{\lambda}{2} \theta \cot \left(\frac{1}{2} \theta\right) d \theta=G_{1}(\lambda / 2), \\
J_{\lambda+1}^{(1)}+J_{\lambda-1}^{(1)} & =2 \ln (2)+\int_{0}^{\pi / 2}\left(1-\cos \frac{\lambda}{2} \theta\right) \cot \frac{1}{2} \theta d \theta \\
& =2 \ln (2)+G_{2}(\lambda / 2),
\end{aligned}
$$

where the integrals

$$
G_{1}(\alpha)=\int_{0}^{\pi} \sin \alpha \theta \cot \frac{1}{2} \theta d \theta \quad \text { and } \quad G_{2}(\alpha)=\int_{0}^{\pi}(1-\cos \alpha \theta) \cot \frac{1}{2} \theta d \theta
$$

can be evaluated by a limiting process from the known result $[2$, Chapter I, p. 8] :

$$
P(x, y)+i Q(x, y)=\int_{0}^{\pi} \sin ^{x} t e^{i y t} d t=\frac{\pi}{2^{x}}\left[\frac{\Gamma(1+x) e^{i \pi y / 2}}{\Gamma\left(1+\frac{x+y}{2}\right) \Gamma\left(1+\frac{x-y}{2}\right)}\right]
$$

We illustrate the procedure for $G_{1}$, which, since $\cot 1 / 2 \theta=(1+\cos \theta) / \sin \theta$, can be written

$$
\begin{aligned}
G_{1}(\alpha) & =\frac{1}{2} \int_{0}^{\pi} \frac{2 \sin \alpha \theta+\sin (\alpha+1) \theta+\sin (\alpha-1) \theta}{\sin \theta} d \theta \\
& =\frac{1}{2} \lim _{x \rightarrow-1}[2 Q(x, \alpha)+Q(x, \alpha+1)+Q(x, \alpha-1)]
\end{aligned}
$$

After substituting from (14), combining, and simplifying with the aid of known $\Gamma$-function identities, this becomes

$$
\begin{array}{r}
G_{1}(\alpha)=\lim _{x \rightarrow-1} \pi(1+x)^{-1}\left[\frac{2 \sin \frac{\pi}{2} \alpha}{\Gamma\left(1+\frac{x+\alpha}{2}\right) \Gamma\left(1+\frac{x-\alpha}{2}\right)}\right. \\
\left.-\frac{\alpha \cos \frac{\pi}{2} \alpha}{\Gamma\left(\frac{3}{2}+\frac{x+\alpha}{2}\right) \Gamma\left(\frac{3}{2}+\frac{x-\alpha}{2}\right)}\right]
\end{array}
$$

The limit is easily evaluated with the aid of l'Hospital's rule, and, upon making use of various familiar identities relating to the $\Gamma$ - and $\Psi$-functions, we get ultimately

$$
G_{1}(\alpha)=\pi-\frac{\sin \pi \alpha}{\alpha}\left\{1+\alpha\left[\Psi\left(\frac{1+\alpha}{2}\right)-\Psi\left(1+\frac{\alpha}{2}\right)\right]\right\}
$$

In an exactly analogous way we find*

(18) $G_{2}(\alpha)=-2 \Psi\left(\frac{1}{2}\right)-\frac{1-\cos \pi \alpha}{\alpha}+2\left[\cos ^{2} \frac{\pi}{2} \alpha \Psi\left(\frac{1+\alpha}{2}\right)+\sin ^{2} \frac{\pi}{2} \alpha \Psi\left(1+\frac{\alpha}{2}\right)\right]$,

and, when (17) and (18) are substituted into (12), the results combined with (3) and (4), and certain obvious simplifications made, expressions identical to (11) are obtained. 
As an exercise, the reader may verify that, when $\lambda$ is an integer, (11) reduces to the previously obtained relations (5) and (6). In this regard, the numerous identities dealing with the $\Psi$-function of rational argument found in [3, Chapter XXIV] will prove helpful.

5. The Integrals $I_{\lambda}^{(2)}=\int_{0}^{\pi / 2}(\sin \lambda \theta / \sin \theta)^{2} d \theta, J_{\lambda}^{(2)}=\int_{0}^{\pi / 2}((1-\cos \lambda \theta) / \sin \theta)^{2} d \theta$. The first of these integrals, according to [1, formula $3.624(6)$ is equal to

$$
\frac{\lambda \pi}{2}
$$

Although it is not so stated in the above reference, this result only holds if $\lambda$ is an integer. For noninteger values of $\lambda$, the integral can be evaluated in the following way:

We have

$$
I_{\lambda}^{(2)} \equiv \int_{0}^{\pi / 2}\left(\frac{\sin \lambda \theta}{\sin \theta}\right)^{2} d \theta=\frac{1}{2} \int_{0}^{\pi i 1-\cos \lambda \phi} \frac{1-\cos \phi}{1-}
$$

Consider the more general integral

$$
\int_{0}^{\pi} \frac{1-\cos \lambda \phi}{\cosh \alpha-\cos \phi} d \phi=\frac{\pi}{\sinh \alpha}-\int_{0}^{\pi} \frac{\cos \lambda \phi}{\cosh \alpha-\cos \phi} d \phi
$$

and let

$$
y(\lambda, \alpha)=\sinh \alpha \int_{0}^{\pi} \frac{\cos \lambda \phi}{\cosh \alpha-\cos \phi} d \phi
$$

so that

$$
\int_{0}^{\pi} \frac{1-\cos \lambda \phi}{\cosh \alpha-\cos \phi} d \phi=\frac{\pi-y(\lambda, \alpha)}{\sinh \alpha}
$$

Now, differentiation of $(22)$ with respect to " $\alpha$ ", followed by integration by parts, gives

$$
\frac{\partial y}{\partial \alpha}=-\lambda \int_{0}^{\pi} \frac{\sin \lambda \phi \sin \phi}{\cosh \alpha-\cos \phi} d \phi
$$

Hence, since

$$
\int_{0}^{\pi} \frac{1-\cos \lambda \phi}{1-\cos \phi} d \phi=\lim _{\alpha \rightarrow 0}\left(\frac{1-y(\lambda, \alpha)}{\sinh \alpha}\right)
$$

application of l'Hospital's rule gives

$$
\int_{0}^{\pi} \frac{1-\cos \lambda \phi}{1-\cos \phi} d \phi=\lambda \int_{0}^{\pi} \frac{\sin \lambda \phi \sin \phi}{1-\cos \phi} d \phi=\lambda G_{1}(\lambda) .
$$

Substituting the expression for $G_{1}(\lambda)$ from (17):

$$
\int_{0}^{\pi / 2}\left(\frac{\sin \lambda \theta}{\sin \theta}\right)^{2} d \theta=\frac{\lambda \pi}{2}-\frac{\sin \lambda \pi}{2}\left\{1+\lambda\left[\Psi\left(\frac{1+\lambda}{2}\right)-\Psi\left(1+\frac{\lambda}{2}\right)\right]\right\}
$$


and since

$$
\int_{0}^{\pi / 2}\left(\frac{1-\cos \lambda \theta}{\sin \theta}\right)^{2}=4 \cdot \int_{0}^{\pi / 2}\left(\frac{\sin \frac{\lambda}{2} \theta}{\sin \theta}\right)^{2} d \theta-\int_{0}^{\pi / 2}\left(\frac{\sin \lambda \theta}{\sin \theta}\right)^{2}
$$

this integral can be expressed in a similar fashion.

6. As a final observation, it may be mentioned that integrals of the type

$$
\int_{0}^{\pi / 2 N} \frac{\sin \lambda \theta}{\sin \theta} d \theta
$$

and

$$
\int_{0}^{\pi / 2 N} \frac{1-\cos \lambda \theta}{\sin \theta} d \theta
$$

where $N$ is an integer, can be expressed as finite combinations of the integrals $I_{\lambda}^{(1)}$ and $J_{\lambda}^{(1)}$ with the aid of the relations

(31) $\frac{\sin 2 M \theta}{\sin \theta}=2 \sum_{n=1}^{M} \cos (2 n-1) \theta, \frac{\sin (2 M+1) \theta}{\sin \theta}=1+2 \sum_{n=1}^{M} \cos 2 n \theta$,

after making the change of variable $\theta$ to $\phi / N$, giving

$$
\begin{aligned}
\int_{0}^{\pi / 2 N} \frac{\sin \lambda \theta}{\sin \theta} d \theta & =\frac{2}{N} \int_{0}^{\pi / 2} \frac{\sin (\lambda \phi / N)}{\sin \phi}\left[\sum_{n=1}^{N / 2} \cos \frac{(2 n-1) \phi}{N}\right] d \phi, \quad N \text { even } \\
& =\frac{1}{N} \int_{0}^{\pi / 2} \frac{\sin (\lambda \phi / N)}{\sin \phi}\left[1+2 \sum_{n=1}^{(N-1) / 2} \cos \frac{2 n \phi}{N}\right] d \phi, \quad N \text { odd }
\end{aligned}
$$

with similar expressions resulting for

$$
\int_{0}^{\pi / 2 N} \frac{1-\cos \lambda \theta}{\sin \theta} d \theta
$$

1885 California, Apt. 62

Mountain View, California 94041

1. I. S. GRADSHTEYN \& I. M. RYZHIK, Table of Integrals, Series and Products, Academic Press, New York, 1965.

2. W. MAGNUS \& F. OBERHETTINGER, Formeln und Sätze fur die Speziellen Funktionen der Mathematischen Physik, Springer-Verlag, Berlin, 1948.

3. J. EDWARDS, A Treatise on the Integral Calculus, Vol. II, Macmillan, New York, 1922; reprinted by Chelsea, New York, 1977. 